\title{
EFFECTS OF SILANE CROSSLINKING ON THERMAL AND MORPHOLOGICAL PROPERTIES OF SPARTIUM JUNCEUM FLOUR/HIGH DENSITY POLYETHYLENE COMPOSITES
}

\author{
Samah IKHLEF, Sorya NEKKAA* and Nacerddine HADDAOUI \\ Laboratoire de Physico-Chimie des Hauts Polymères, Département de Génie des Procédés, Faculté de Technologie, \\ Université Ferhat ABBAS Sétif 1, (19000), Algérie
}

In this study, the use of silane-crosslinking high density polyethylene/Spartium Junceum flour composites has been investigated. The silane grafting and composite production were carried out simultaneously in a one-step process using a mixing chamber of a Plastograph. Extraction in p-xylene revealed an increase in gel content with crosslinking. Therefore, storage in a high humidity sauna generated a higher degree of crosslinking in the composites than storage at room temperature. The thermal stability of the composite is greatly improved by crosslinking reaction, but it isn't affected by the vinyltrimethoxysilane crosslinker concentration. Differential scanning calorimetry measurements of the composites showed a lower crystallinity in the crosslinked samples as compared to the uncrosslinked ones.

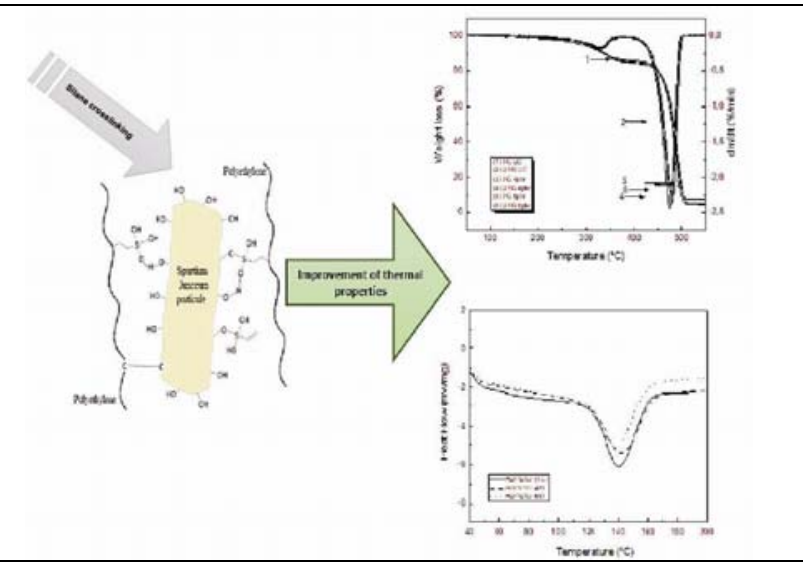

\section{INTRODUCTION}

Natural fiber reinforced polymer composites are being currently used to reinforce the polymer matrix in order to improve its mechanical properties. These are environmental friendly and cost effective compared to synthetic fiber reinforced composites. The availability of natural fiber, their low cost and ease of manufacturing have urged researchers worldwide to try locally available inexpensive fiber and to study their feasibility for reinforcement purposes. They satisfy to some excess the required specifications of good reinforced polymer composite for industrial and structural applications. ${ }^{1,2}$ Natural fibers need to be treated chemically in order to improve interfacial adhesion between fiber surface and polymer matrix. The chemically treated natural fibers show better improvement in properties than the untreated fibers. This can be attributed to the removal of waxy layer from the surface of natural fibers, which make them rougher. Natural fiber reinforcements have resulted in improved impact toughness and fatigue strength. Many efforts have been made by researchers towards improving mechanical properties, directed at improving the interface between fiber and polymer.,

Several strategies of chemical treatment aiming at improving the compatibility between natural fibers and polymer matrix and their interfacial adhesion... ${ }^{2,4}$ The means of improving the interfacial adhesion properties is by creating chemical crosslinks between the fiber and the polymer matrix as well as within the matrix using

\footnotetext{
*Corresponding author: s_nekkaa@yahoo.fr; snekkaa@univ-setif.dz
} 
silane crosslinking method. ${ }^{5,6}$ The objective of this work is to investigate the influence of silanecrosslinking of high density polyethylene/Spartium junceum composites and its effect on gel content, swell ratio, thermal and morphological proprieties of polyethylene/Spartium junceum composites. The crosslinked composites were produced using a high and a low silane-solution (VTMS: DCP).

\section{RESULTS AND DISCUSSION}

The basic principle of silane-crosslinking is to graft the silane onto the backbone of the polyethylene chain. This grafting takes place in a reactive extrusion process, where a solution of a vinyl-alkoxysilane and peroxide is compounded with polyethylene. The peroxide decomposes and leaves oxy-radicals which attract hydrogen from the polyethylene chains to create radical sites. Thus, the vinyl-group of the alkoxysilane opens and grafts to the polyethylene chain. As water is diffused into the plastic afterwards, the grafted alkoxysilanes undergo hydrolysis to form silanols, and a condensation reaction then forms siloxanebridges, as displayed in Figure 1. ${ }^{7}$

(1)

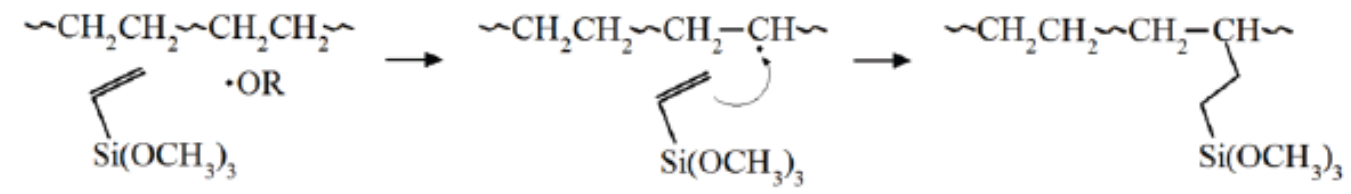

(2)<smiles>CCCC(C)CCC(CCC(C)CC)CC(C)O</smiles>

Fig. 1 - The hydrolysis step (1) and condensation step (2) during silane crosslinking. ${ }^{7}$

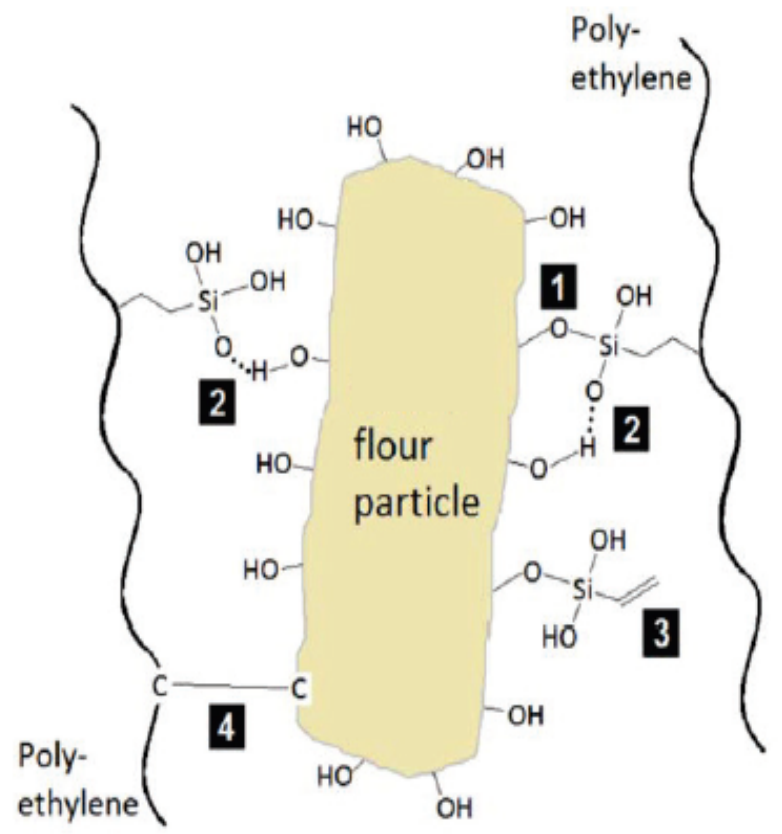

Fig. 2 - Previous suggested mechanisms. 1) -Si-O-C-, 2) Hydrogen bonds,

3) Van der Waals forces between the silanes condensed to flour but not grafted to PE, 4) free radical reaction, -C-C-. ${ }^{5}$ 
The degree and rate of formed silane-crosslinks is affected by the storage environment of the composite. If the composite is kept in a high temperature and a humid environment, more water will access the grafted alkoxysilanes and the probability of hydrolysis and condensation is higher. This may theoretically lead to a higher degree and rate of crosslinking. A high temperature leads to a higher free-volume of the polyethylene and a higher content of water which is carried by the air leading to a greater flux into the material. ${ }^{7}$ For silane-crosslinked natural composites, an important improvement in strength and toughness is observed. This indicates that chemical links between the natural fiber and the polymer took place. It is believed that these links are a mix of covalent bonding ( $\mathrm{Si}-\mathrm{O}-\mathrm{C}$ bridges, $\mathrm{C}-\mathrm{C}$ crosslinks), hydrogen bonding and Van der Waals forces witch are displayed in Figure 2.

\section{Gel content (GC)}

The degree of crosslinking in the composites was determined by gel content measurements. Crosslinked polyethylene is insoluble in boiling pxylene while the non-crosslinked part is soluble. The gel content thus corresponds to the crosslinked part of the polyethylene and is determined by calculating the weight loss during p-xylene extraction. In crosslinking reactions, peroxide initiates the reaction at elevated temperature and form free radicals. Free radicals of peroxide will extract hydrogen from the polyethylene molecules, thus creating unstable carbon. After which, silane is grafted onto the carbon to form a copolymer and the remaining free radical on the copolymer branch will continue to abstract a hydrogen atom from other polyethylene molecule to become stable. ${ }^{8}$ Which results, it found that the gel content of HDPE/4 was $20 \%$ and $21 \%$ for HDPE $/ 6$, when it was $45 \%$ for HDPE/SJ/4 composite and $49 \%$ for $\mathrm{HDPE} / \mathrm{SJ} / 6$ composite. It has been found that the gel content increases with increasing crosslinker concentration and flour content. Also, from a previous study ${ }^{9,10}$ with silane grafted HDPE, it was shown that fiber could be incorporated in the crosslink network since the silanol groups react with hydroxyl groups of the fiber as well as with other hydroxyl groups grafted on the polyethylene backbone.

The basic principle of silane-crosslinking is to graft vinyl silane onto polyethylene or filler chains. The peroxide first decomposes and creates oxy radicals, which have the potential to abstract hydrogen from the polyethylene or filler polymer but can also attack the vinyl group of the VTMS molecule and convert it into radicals. These free radicals either combine with one another or attack another molecule in the same fashion to propagate the free-radical reaction. As water diffused into the plastic afterwards, the grafted alkoxysilanes undergo hydrolysis to form silanols, and a condensation reaction then forms siloxane-bridges. ${ }^{5,7}$

The degree and rate of formed silane-crosslinks is affected by the environment where the composite is stored. If the composite is kept in a high temperature and humid environment, more water will access the grafted alkoxysilanes and the probability of hydrolysis and condensation is higher. The higher temperatures lead to higher free-volume of the polyethylene and to higher content of water carried by the air leading to more water transported into the material. ${ }^{7,11,12}$ This is confirmed by the improvement of gel content after storage in a sauna. It was 39\% for HDPE $/ 4$ and $43 \%$ for HDPE/ 6 when it was $63 \%$ for $\mathrm{HDPE} / \mathrm{SJ} / 4$ composite and $71 \%$ for $\mathrm{HDPE} / \mathrm{SJ} / 4$ composite after storage at sauna.

The high degree of crosslinking from the sauna storage induces a higher proportions of amorphous phase in the thermoplastic matrix thus, facilitating the condensation of silanols for the formation of crosslinks. In addition, higher temperatures rendered it possible for the air to carry more water, a positive point for such moisture-induced crosslinking. ${ }^{5}$ Formation of a network with covalent bonds between flour and polyethylene would improve the interfacial adhesion and improve the mechanical properties of the composites. Crosslinking could also lead to a stabilization of the morphology improving the longterm properties compared to non-crosslinked composites. Moreover, crosslinked composites also give the possibility of utilizing mixtures of polymeric scrap eventually leading to useful materials. ${ }^{10}$

\section{Swell ratio}

From Figure 3, one can notice that the addition of the silane solution results in a reduction in the swelling ratio. Thus, the addition of silane increases the degree of crosslinking. Moreover, storage in sauna increases significantly the gel content and decreases the swelling process of crosslinked composites. Furthermore, it is as shown in Figure 3 that there is a linear relationship between the degree of crosslinking and the swelling ratio. The network density increases with the increase of gel content, so composites stored in the sauna shows the highest network density. Our results are in accordance with the findings of Bengtsson and Oksman. ${ }^{13}$ 


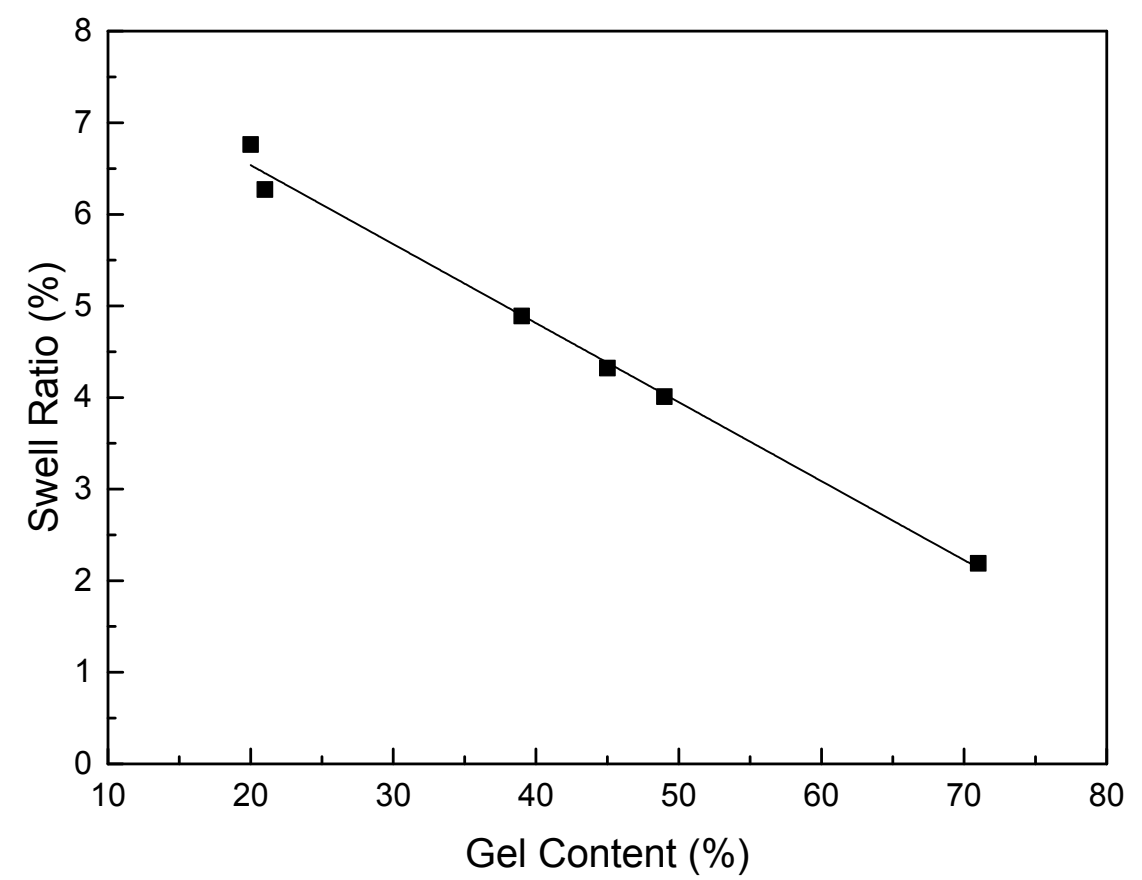

Fig. 3 - Swell ratio as a function of gel content in the crosslinked HDPE/Spartium junceum $30 \mathrm{wt} \%$ composites.

\section{Thermogravimetric analysis (TGA)}

From Figure 4, it reveals that the onset temperature of thermal degradation increased in crosslinked composites. The thermal stability of composite is not greatly improved by the crosslinking reaction. ${ }^{14}$ The values of the onset temperature of thermal degradation showed that the thermal stability of crosslinked composites is not affect by the VTMS crosslinker concentration. ${ }^{8}$ On the other hand, the temperature of maximal thermal degradation increased as VTMS crosslinker concentration is increased $\left(T_{d} \max\right.$, untreated $487^{\circ} \mathrm{C} .4 \mathrm{pcr} 489^{\circ} \mathrm{C}$. $6 \mathrm{pcr} 495^{\circ} \mathrm{C}$ ).

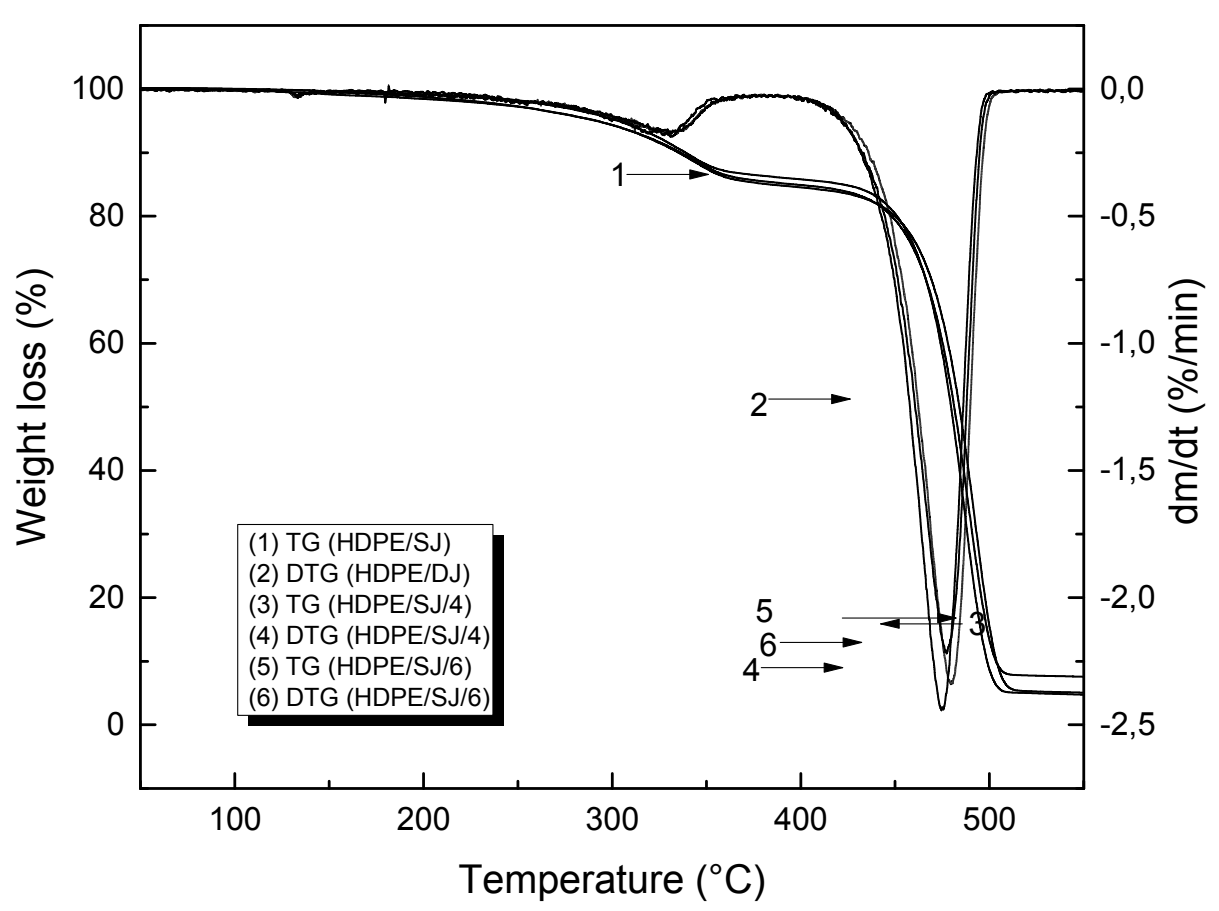

Fig. 4 - TG and DTG curves of uncrosslinked and crosslinked HDPE/ Spartium junceum $30 \mathrm{wt} \%$ composites. 
During silane treatment, various type of reactions take place at the surface of the Spartium junceum composites flour, such as hydrolysis, condensation, hydrogen bonding and covalent bond formation. Silanol molecules react with the hydroxyl group of the fiber resulting in formation of strong covalent bonds to the cell wall. ${ }^{15}$ Also the free silanols react with each other forming -Si-O-Si- bond. The vinyl group of the silane molecule couples with the thermoplastic matrix and increases the physical compatibility. The organo-functional group of the silane molecule (vinyl) couples with the matrix and increases the strength of bonding. The enhanced covalent bonding added with physical compatibility with the polymer matrix increases the thermal stability of the resultant composites. ${ }^{16}$ So the crosslinking reaction can raise the limit of composite in practical application.

\section{Differential scanning calorimetry (DSC)}

DSC was used to investigate the influence of the filler and the crosslinking on the melting temperature, and the specific enthalpy of melting. The results obtained from the DSC analysis are summarized in Table 1 . The degree of crystallinity, $\chi_{c}$, of the samples is calculated according to the Equation:

$$
X_{c}(\%)=\frac{\Delta H m}{\Delta H \infty} \times \frac{100}{w}
$$

where $\Delta H_{\infty}$ is the melting enthalpy of $100 \%$ crystalline polymer, was taken as $290 \mathrm{~J} \mathrm{~g}^{-}{ }^{1}$ for PE. $\Delta H_{m}$ is the enthalpy of fusion, and (w) is the weight fraction of the polymer matrix material in the composites.

Figure 5 shows the heating DSC thermographs of uncrosslinked and crosslinked composites stored at room temperature. The degree of crystallinity, enthalpy of melting and melting peak temperatures from the DSC analyses are summarized in Table 1. It is observed that changes in the thermal characteristics are more prononced when HDPE/ Spartium junceum flour composites was crosslinked. Decreases in the melting temperature and enthalpy were observed because crosslinking reduces the polyolefin crystallinity. Crosslinks act as defect centers, which impeach the folding of macromolecular chains and decrease the size of lamellar crystals. The reduction in the lamellar thickness of the crystallites leads to a decrease in the melting temperature $\left(\mathrm{T}_{\mathrm{m}}\right)$, which is also true for the crystallization. ${ }^{17,18}$

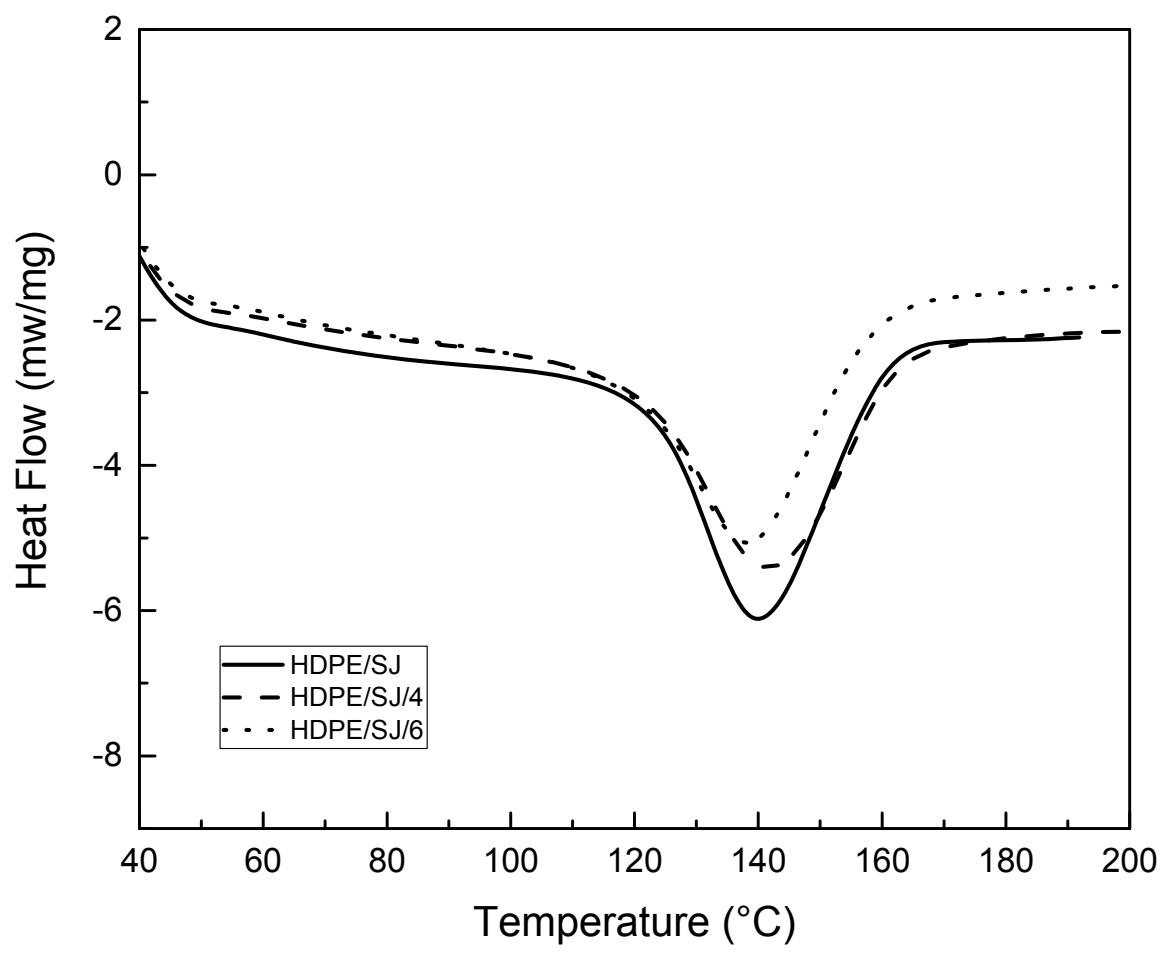

Fig. 5 - DSC curves of crosslinked and uncrosslinked HDPE/ Spartium junceum $30 \mathrm{wt} \%$ composites. 
Table 1

Influence of crosslinking on the thermal properties of HDPE and HDPE/ Spartium junceum flour composites

\begin{tabular}{l|l|l|l}
\hline Samples & $\mathbf{T}_{\mathbf{m}}\left({ }^{\circ} \mathbf{C}\right)$ & $\Delta \mathbf{H}_{\mathbf{m}}(\mathbf{J} / \mathbf{g})$ & $\mathrm{x}_{\mathbf{c}}(\mathbf{\%})$ \\
\hline HDPE & 141 & 221 & 76 \\
HDPE/4 & 146 & 215 & 74 \\
HDPE/6 & 141 & 214 & 74 \\
HDPE/SJ & 140 & 204 & 70 \\
HDPE/SJ/4 & 142 & 190 & 66 \\
HDPE/SJ/6 & 138 & 180 & 62 \\
\hline
\end{tabular}

Figure 5 show that the crosslinked composites shows a lower melting endotherm than the uncrosslinked ones. This is corroborated by the low degree of crystallinity in observed the crosslinked composites. This means that as the crosslinked density increases, the degree of crystallinity and crystallite thickness decreases. Reduction in crystallinity occurs because of crosslinking tacking place in amorphous phase. There is also some breakdown of crystallinity. Meaning that the crosslinking reaction decreases the mobility of chains matrix. The melting peak temperature of the crosslinked composites is also slightly lower than in the uncrosslinked ones. It was also further confirmed from the results obtained by previous studies. ${ }^{7,13,15}$

\section{Atomic force microscopy (AFM)}

From Figure 6, we can observe that the surface of uncrosslinked composites shows an irregular topography and voids between the filler and the matrix. Also, the filler bonding appears as clear spots on a dark cavities of various size at the interface between the cellulose filler and the HDPE matrix, which creates more irregularities in the surface. ${ }^{19-21}$ This is mainly due to the poor adhesion between the Spartium junceum flour and the HDPE matrix.

The AFM micrographs illustrate a reduction of surface roughness in crosslinked composites compared to the uncrosslinked ones, as shown in Figures 7 and 8 . The roughness value of uncrosslinked composites is $35.628 \mathrm{~nm}$ when in crosslinked composites ( $4 \mathrm{phr}$ and $6 \mathrm{phr}$ ) this value is 23.932 and $28.892 \mathrm{~nm}$, respectively. However, the contact area was much larger and no dark cavities were observed in the case of crosslinking composites, suggesting a much stronger interfacial adhesion between Spartium junceum flour and HDPE matrix. These observations suggested a stronger interfacial adhesion, created by crosslinking that will allow more efficient load transfer. These findings explain the increases noticed in the tensile strength and the elongation at break of the crosslinked composites with respect to uncrosslinked ones.
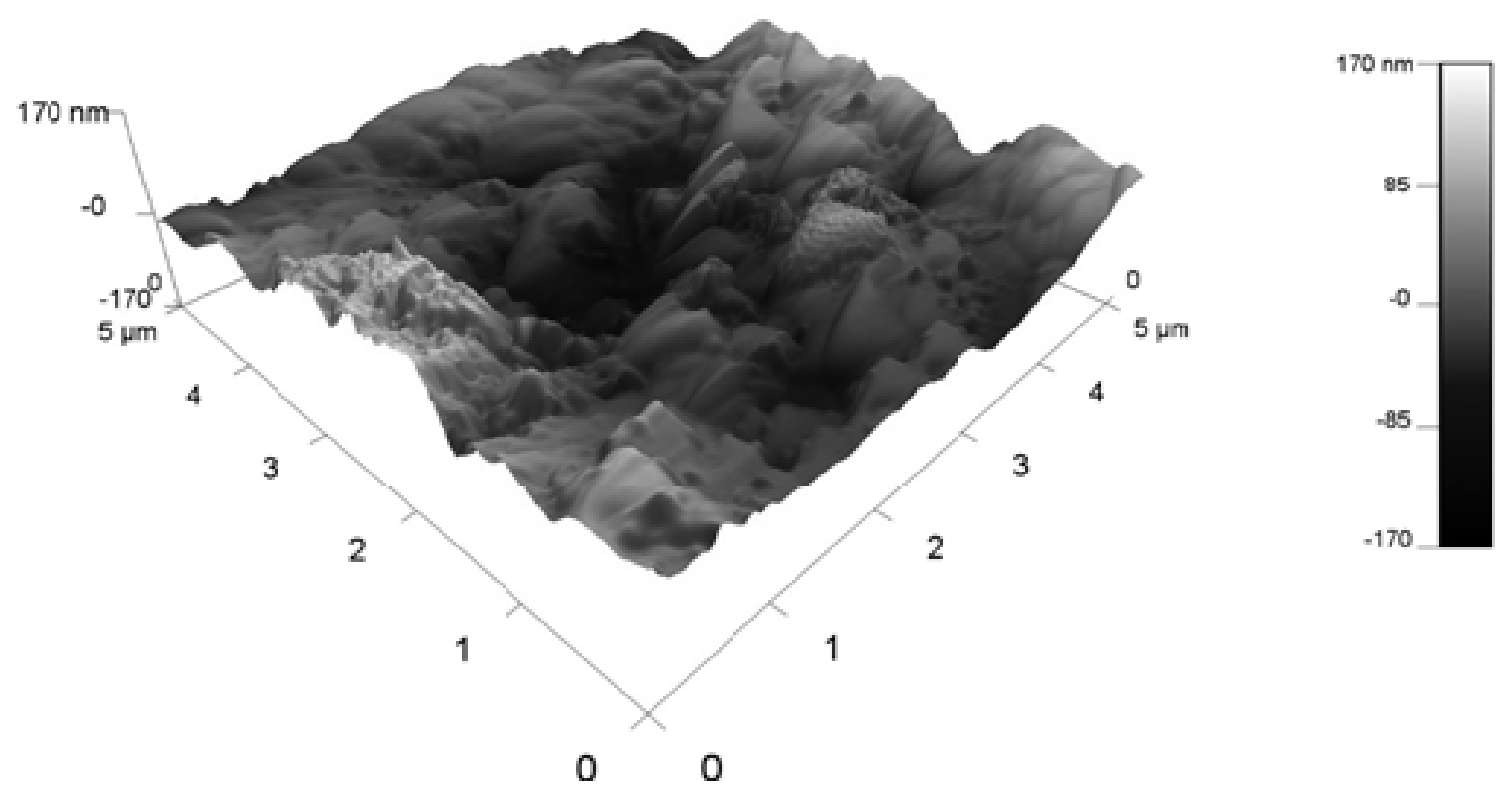

Fig. 6 - AFM topographic image of uncrosslinked HDPE/Spartium junceum $30 \mathrm{wt} \%$ composite. 


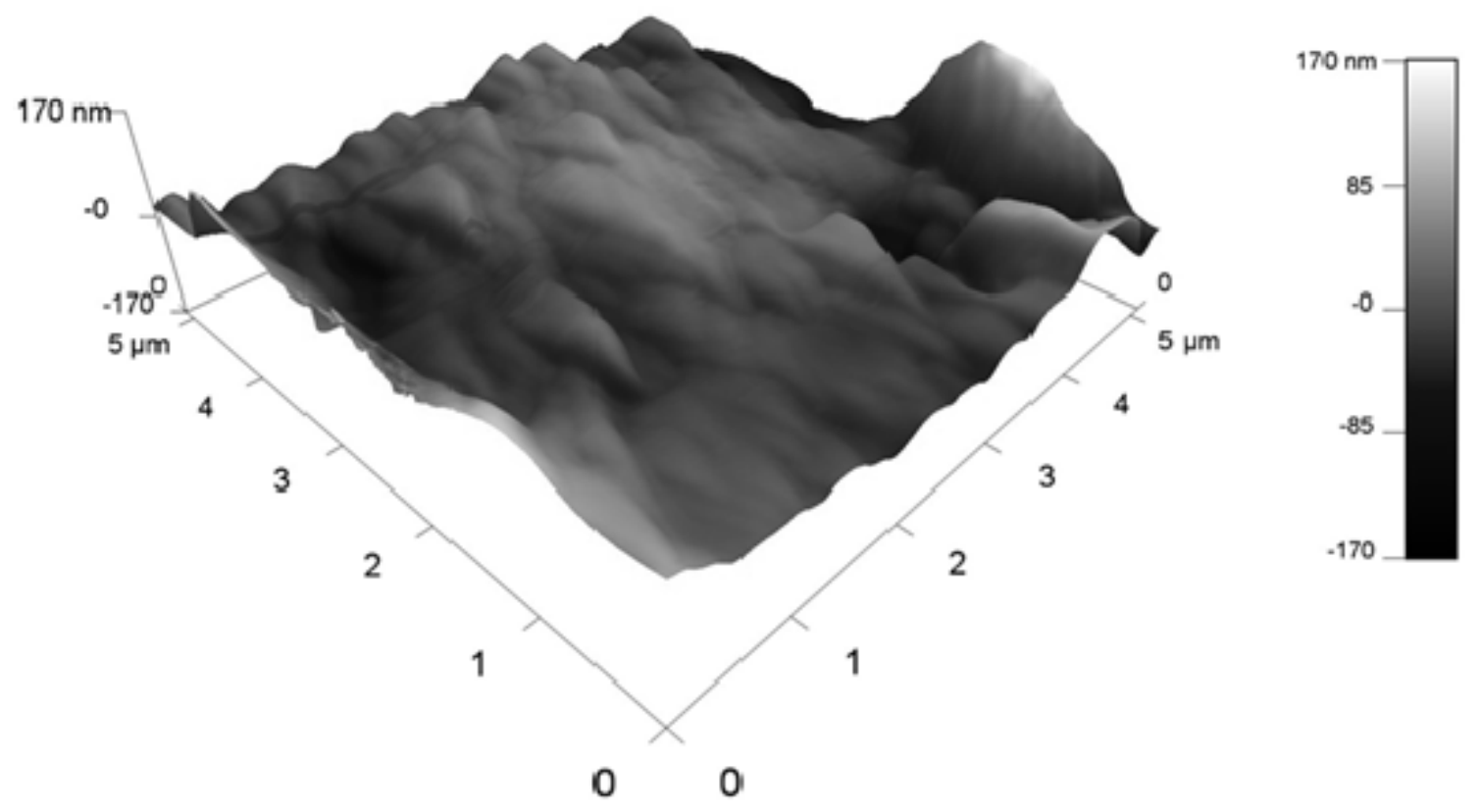

Fig. 7 - AFM topographic image of crosslinked (4phr) HDPE/Spartium junceum $30 \mathrm{wt} \%$ composite.
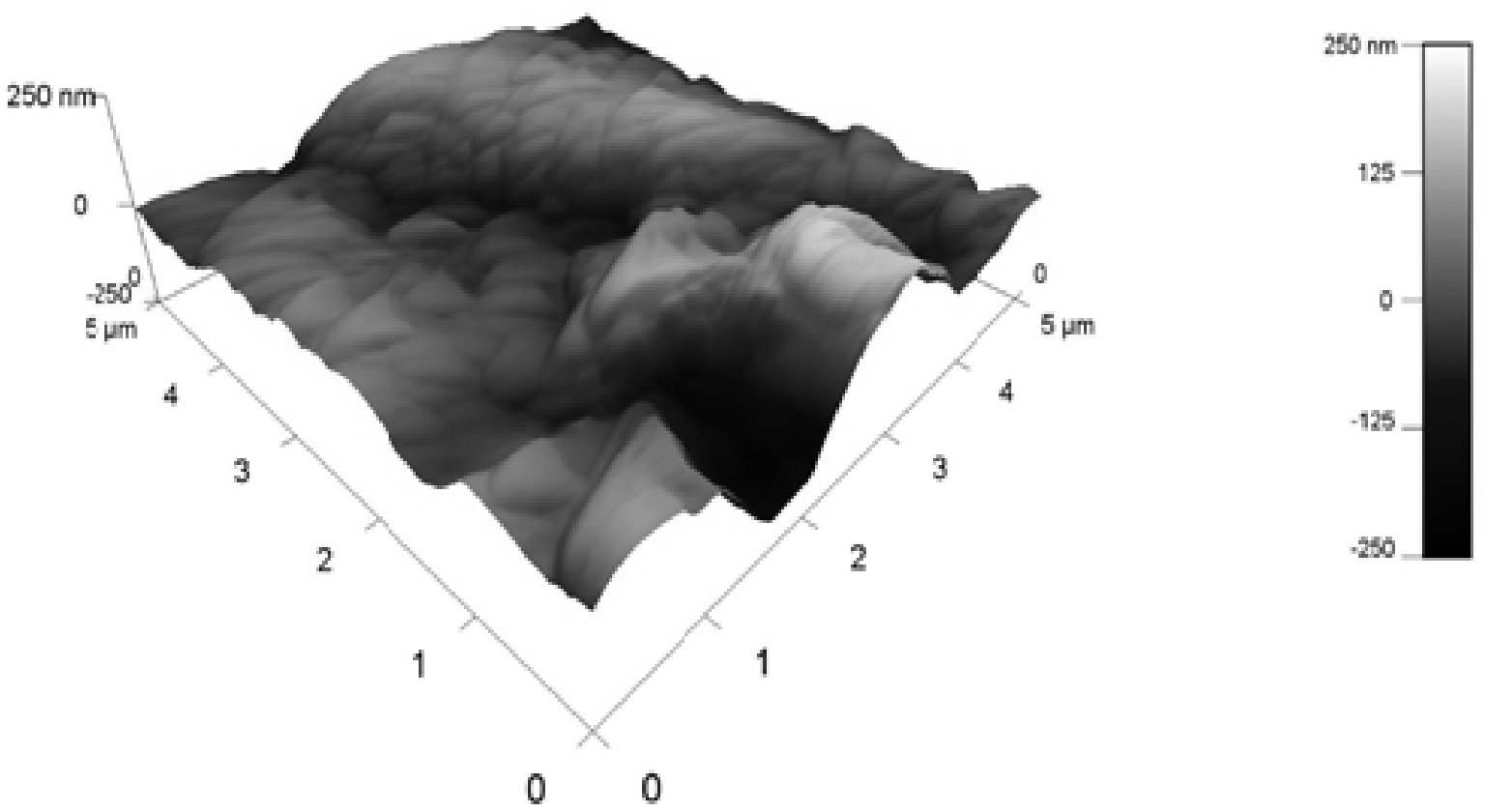

\section{EXPERIMENTAL AND METHODS}

\section{Materials and methods}

The polymer matrix is a high density polyethylene (HDPE) M80064 with a melt flow index of 8 (g/10 min, 2.16 $\mathrm{kg} / 190{ }^{\circ} \mathrm{C}$ ), produced by ExxonMobil. The filler used in this work is Spartium junceum. The stems of plant were cut into 8 to $12 \mathrm{~cm}$ pieces and air-dried for 48 hours and then oven-dried at $70^{\circ} \mathrm{C}$ for $24 \mathrm{~h}$. The obtained filler pieces were then reduced by grinding in order to obtain a very fine flour. The latter is sifted in order to separate a grain size flour between 100 and $200 \mu \mathrm{m}$. The flour obtained was further washed with distilled water in the presence of a detergent and a few drops of bleach for $2 \mathrm{~h}$ at $50^{\circ} \mathrm{C}$. Then it is pretreatment with $\mathrm{NaOH}(1 \mathrm{wt} \%)$ for $2 \mathrm{~h}$ to remove the extractables and followed by neutralization with acetic acid ( $1 \mathrm{wt} \%)$ for 15 minutes, the $\mathrm{pH}$ of the solution was 7. Furthermore, the flour is filtered and washed three times with distilled water. Subsequently, the flour is dried for $24 \mathrm{~h}$ at $60{ }^{\circ} \mathrm{C}$. A stearic acid was used as a lubricant to improve the surface quality. The reactants for crosslinking were vinyltrimethoxy silane VTMS 97\%, and dicumyl-peroxide DCP 98\%, obtained from Sigma Aldrich were used as received.

\section{Processing}

The HDPE/ Spartium junceum composites were prepared via melt mixing a mixing chamber of a Plastograph at $150^{\circ} \mathrm{C}$, $30 \mathrm{rpm}$ and $10 \mathrm{~min}$. The composites were prepared with 30 
wt $\%$ of reinforcement. Silane crosslinked composites were produced by adding a solution of vinyltrimethoxysilane and dicumyl peroxide to the mixture two minutes before the end of the mixing. The mixing ratios of silane and peroxide $(\mathrm{w} / \mathrm{w})$ were 12:1. The crosslinked composites were produced using a high and low silane-solution (VTMS: DCP) $6 \mathrm{phr}$ and $4 \mathrm{phr}$ content. The melt pressing of the prepared samples was performed at $180^{\circ} \mathrm{C}$ and $150 \mathrm{~kg} / \mathrm{cm}^{2}$. After processing, the specimens were stored at sauna at $100 \% \mathrm{RH}$ and $90^{\circ} \mathrm{C}$. The sauna-stored samples were subsequently dried to their initial weight before testing.

\section{Techniques \\ Gel content}

The degree of crosslinking of silane crosslinked samples was measured by determining their gel content after the crosslinking reactions. The determination of the gel content test was performed via p-xylene extraction technique containing $1 \mathrm{wt} \%$ antioxidant (Butylated hydroxytoluene BHT) at $140^{\circ}$ for 24 hours.

$$
\text { Gel content }(\%)=100-\frac{\text { weight lost during extraction }}{\text { weight of original specimen }- \text { weight of filler }}
$$

\section{Swell ratio}

The specimens were extracted in one piece using p-xylene as solvent at temperature of $110^{\circ} \mathrm{C}$ for 24 hours. The swell ratio of the samples was calculated as follows:

$$
\text { Swell ratio }(\%)=\frac{w_{g}-w_{d}}{w_{o}-w_{e}} K+1
$$

$\mathrm{W}_{\mathrm{g}}$ : the weight of swollen gel after the immersion period; $\mathrm{W}_{\mathrm{d}}$ : the weight of dried gel;

$\mathrm{W}_{\mathrm{o}}=f \mathrm{~W}_{\mathrm{s}}$; When $f$ is the ratio of the weight of the polymer in the formulation to the total weight of the formulation;

$\mathrm{W}_{\mathrm{s}}$ : the weight of specimen being tested;

$\mathrm{W}_{\mathrm{e}}$ : the weight of extract;

$\mathrm{K}$ : the ratio of the density of HDPE to that of the solvent at $110{ }^{\circ} \mathrm{C}$ and is equal to 1.17

\section{Thermogravimetric analysis}

The thermal decomposition of composites and filler was evaluated by thermogravimetric analysis (TGA) using a Perkin Elmer TGA 4000 instrument. Sample was heated under oxidation from room temperature to $550^{\circ} \mathrm{C}$ at a rate of $10^{\circ} \mathrm{C} / \mathrm{min}$.

\section{Differential scanning calorimetry (DSC)}

The crystallization and melting behavior of the samples before and after silane crosslinking was analyzed using a differential scanning calorimeter (NETZSCH DSC 214). The test specimens $(15 \mathrm{mg})$ were heated from 20 to $300^{\circ} \mathrm{C}$ at a scan rate of $10^{\circ} \mathrm{C} / \mathrm{min}$, under a nitrogen atmosphere (a purge rate of $20 \mathrm{~cm}^{3} / \mathrm{min}$ )

\section{Atomic force microscope}

The surface topography of the composites was investigated using Asulym Research-Model MFP-3D Atomic force microscope (AFM), with ambient conditions and resonance frequency of $1 \mathrm{~Hz}$. Root mean square (RMS) roughness data were obtained by analyzing topography micrograhs using the size of $20 \mu \mathrm{m} \times 20 \mu \mathrm{m}$ specimens.

\section{CONCLUSION}

This study focused on process optimization and evaluation of thermal and structural properties of silane crosslinked composites at different concentration. Gel content was significantly increased after storage in a sauna when the swelling ratio of the crosslinked composites was decreased. Crosslinking reaction shifts the onset temperature of thermal degradation of composites to higher values. On the other hand, the crosslinking reaction increase the temperature of maximal thermal degradation and decreases the crystallinity.

Table 2

Abbreviations used in the results and discussions section

\begin{tabular}{l|l}
\hline Formulation & Abbreviation \\
\hline uncrosslinked HDPE & HDPE \\
crosslinked HDPE with 4phr of crosslinker (VTMS:DCP)(12:1) & HDPE/4 \\
crosslinked HDPE with 6phr of crosslinker (VTMS:DCP)(12:1) & HDPE/6 \\
uncrosslinked HDPE/SJ composite & HDPE/SJ \\
crosslinked HDPE/SJ composite with 4phr of crosslinker (VTMS:DCP)(12:1) & HDPE/SJ/4 \\
crosslinked HDPE/SJ composite with 4phr of crosslinker (VTMS:DCP)(12:1) & HDPE/SJ/6 \\
\hline
\end{tabular}




\section{REFERENCES}

1. Y. Habibi, W.K. El-Zawawy, M.M. Ibrahim, M.M. Ibrahim and A. Dufresne, Compos. Sci. Technol., 2008, 68, 18771885.

2. F.Z. Arrakhiz, M. El Achaby, M. Malha, M.O. Bensalah, O. Fassi-Fehri and R. Bouhfid, Mater. Des., 2013, 43, 200-205.

3. A.L. Bhongade and S.P. Borkar, Int. J. Adv. Eng. Res. Sci., 2016, 3, 36-39.

4. P. Muensri, T. Kunanopparat, P. Menut and S. Siriwattanayotin, Composites Part A, 2011, 42,173-179.

5. G. Grubbström and K. Oksman, Compos. Sci. Technol., 2009, 69, 1045-1050.

6. K. Sirisinha and S. Chimdist, Polym. Test., 2006, 25, 518-526.

7. M. Bengtsson and K. Oksman, Composites Part A, 2006, 37, 752-765.

8. W.A.W.A., Rahman, N. Adenan, R. Rasit-Ali and H. Sulaiman, J. Appl. Sci., 2009, 9, 3041-3047.

9. E.E.M. Ahmad and A.S. Luyt, Composites Part A, 2012, $43,703-710$.
10. M. Bengtsson, P. Gatenholm, K. Oksman, B. Magnus, G. Paul and O. Kristiina, Compos. Sci. Technol., 2005, $65,1468-1479$.

11. G. Grubbström, A. Holmgren and K. Oksman, Composites Part A, 2010, 41, 678-683.

12. M. Bengtsson, N.M. Stark and K. Oksman, Compos. Sci. Technol., 2007, 67, 2728-2738.

13. C.F. Kuan, H.C. Kuan, C.C.M. Ma and C.M. Huang, Composites Part A, 2006, 37, 1696-1707.

14. A.S. Singha, A.K. Rana and R.K. Jarial, Mater. Des., 2013, 51, 924-934.

15. C.F. Kuan, C.C.M. Ma, H.C. Kuan, H.L. Wu and Y.M. Liao, Compos. Sci. Technol., 2006, 66, 2231-2241.

16. M. Saiful-Islam, S. Hamdan, I. Jusoh, M.R. Rahman and A.S. Ahmed, Mater. Des., 2012, 33, 419-424.

17. C.S. Wu, Polym. Degrad. Stab., 2013, 98, 1473-1480.

18. M. Bengtsson and K. Oksman, Compos. Sci. Technol., 2006, 66, 2177-2186.

19. L.X. Zhong, S.Y. Fu, X.S. Zhou and H.Y. Zhan, Composites Part A, 2011, 42, 244-252.

20. S. Bouhank, S. Nekkaa and N. Haddaoui, J. Adhes. Sci. Technol., 2016, 30, 1462-1478.

21. F.Z. Arrakhiz, M. Elachaby, R. Bouhfid, S. Vaudreuil, M. Essassi and A. Qaiss, Mater. Des., 2012, 35, 318-322. 
